\title{
Delokutivität, Possessive und die italienischen Verwandtschaftsbezeichnungen
}

\author{
Klaus Hölker (Hannover)
}

\begin{abstract}
This contribution is about a hitherto unexplained phenomenon of Italian syntax, i. e. the lack of a determiner in nominal expressions of a certain type that contain a possessive and a kinship noun as its scope, despite the fact that possessives normally require a determiner. So, my car is la mia macchina in Italian, whereas my son translates as mio figlio. It is argued that a plausible solution to this puzzle can be given by using Benveniste's notion of delocutivity. Therefore, the first part of the article presents the concept of delocutivity, mainly drawing on French examples. The main purpose of this introductory part is to show that the class of delocutive expressions is not restricted to verbs.
\end{abstract}

In dem Roman von Lawrence Sterne $A$ Sentimental Journey through France and Italy aus dem Jahr 1768 heißt es an einer Stelle (Die folgenden interessanten Beispiele aus Sterne und Foscolo sind Conte (1984) entnommen.):

(1) Monsieur Dessein had diabled the key above fifty times before he found out he had come with a wrong one in his hand. (Sterne 1948: 47)

Die Form diabled in diesem Satz weist die Eigenschaft auf, die man seit Émile Benvenistes Aufsatz Les verbes délocutifs aus dem Jahr 1958 "Delokutivität" nennt. Was versteht man unter "Delokutivität"? Was ist ein delokutives Verb? Gibt es delokutive Ausdrücke, die anderen syntaktischen Kategorien angehören als der Kategorie der Verben? Ich werde in meinem Beitrag zunächst, vor allem anhand französischer Beispiele, diesen Fragen nachgehen. Es wird sich dabei herausstellen, dass Delokutivität ein morphologisch und semantisch relevantes Phänomen ist, nämlich im Rahmen der Wortbildung. Es überrascht deshalb vielleicht, wenn ich mit Hilfe dieses Konzepts im zweiten Teil des Aufsatzes eine Frage der italienischen Syntax behandle, die m.W. seit Friedrich Diez unbeantwortet ist. Es geht um die Frage, weshalb Italiener, wenn sie von ihrem Auto sprechen, la mia macchina sagen, aber mia moglie, also ohne Artikel, wenn es um ihre Frau geht. Auch für Italienerinnen gilt natürlich, dass sie la mia macchina im ersten Fall sagen und von ihrem Mann als mio marito sprechen. Im allgemeinen gilt im Italienischen, im Gegensatz zum Deutschen, dass in Kombi- 
nation mit einem Possessiv ein Determinator (also z. B. ein Artikel oder ein Demonstrativ) vorkommen muss. Bei bestimmten Verwandtschaftsausdrücken darf unter gewissen Bedingungen aber gerade kein Determinator stehen, wobei der zu erwartende Determinator der bestimmte Artikel ist. *Il mio marito ist also im Standard schlicht ungrammatisch.

\section{Delokutivität}

Kehren wir zunächst zum einleitend zitierten Satz aus Sternes Sentimental Journey zurück. Es ist sicherlich schon klar, worum es geht: diabled ist das Partizip Perfekt eines Verbs * to diable, das es im Lexikon des Englischen gar nicht gibt. Sterne kreiert hier ein Verb aus dem französischen diable ('Teufel'). Das entsprechende englische Wort ist devil. Diesem entspricht im Lexikon tatsächlich das Verb to devil, das aber etwas völlig anderes bedeutet als * to diable im Text von Sterne. To devil bedeutet nämlich: 1. 'stark gepfeffert rösten', 2. (Wolle) 'wolfen', 'krempeln', 3. 'belästigen', 4. 'Hilfsdienste leisten'. Natürlich wäre keine dieser Bedeutungen passend für *to diable, wie es hier vorkommt, so dass man ausschließen kann, dass Sterne vielleicht aus einer Laune heraus an dieser Stelle das gewähltere oder elegantere französische Wort für das englische to devil verwendet. Es handelt sich auch nicht um ein aus dem Französischen entlehntes Verb, also etwa *diabler. Ein solches Verb gibt es im Französischen ebenso wenig wie es * to diable im Englischen gibt. Aber, und das ist der springende Punkt, das Verb *diabler könnte es geben, und es würde verstanden, ebenso wie ein lesender Engländer und eine lesende Engländerin des 18. Jhs *to diable verstehen konnten.

Viel skeptischer, was die Verstehbarkeit jener Neubildung betrifft, war da Sternes italienischer Kollege Ugo Foscolo. Seine Übersetzung des Satzes aus Sterne lautet in Viaggio sentimentale di Yorick lungo la Francia e l'Italia aus dem Jahr 1813 folgendermaßen:

(2) Cinquanta e più diavoli aveva monsieur Dessein chiamati addosso alla chiave prima d'accorgersi che la non era quella della rimessa. (Sterne 1970: 61) ('Fünfzig und mehr Teufel hatte Monsieur Dessein dem Schlüssel an den Hals gewünscht, bevor er merkte, dass es nicht der Schlüssel für den Schuppen war.')

Foscolo liefert hier eine erklärende, eine interpretierende Übersetzung von diabled. Er beschreibt, was Monsieur Dessein tut, wenn er Diable! sagt, und zwar nicht einfach das Substantiv diable äußert, sondern die Fluchformel Diable! (oder auch Que diable! oder Au diable!). Die Übersetzung macht jedoch im Gegensatz zum Original nicht explizit, dass Monsieur Dessein Diable! (Que diable!, Au diable!). geäußert hat. Das kann aus der Übersetzung nur unsicher geschlossen werden, denn der Leser/ die Leserin weiß zwar, dass er Franzose ist, er hätte jedoch auch eine andere Fluchformel verwenden können. 
Nun wollte Foscolo aber offenbar den Lesern und Leserinnen auch nicht die $\mathrm{Fi}$ nesse der Sterneschen Schöpfung diabled vorenthalten. Er liefert nämlich in einer Fußnote eine Übersetzung, in der diabled mit einer italienischen Parallelkonstruktion wiedergegeben wird, und zwar mit diablata. Sicherheitshalber fügt er aber noch einen sprachlichen Kommentar hinzu. Die Fußnote lautet:

(3) Letteralmente: monsieur Dessein aveva diablata la chiave ecc.; dalla esclamazione francese diable! [...] derivò qui il verbo diabled. (Sterne 1970: 61) ('Wörtlich: Monsieur Dessein hatte den Schlüssel diablet/gediablet (!) usw.; aus dem französischen Ausruf diable! [...] hat er hier das Verb diabled abgeleitet.')

Diablata ist Femininum Singular des Partizips Perfekt eines im italienischen Lexikon nicht vorhandenen *diablare.

Es ist also klar, dass es bei dieser Neuschöpfung ganz wichtig ist, dass nicht einfach das französische Wort für 'Teufel', also diable, Basis des Verbs * to diable ist, sondern die Fluchformel Diable!. Ein *diavolare, das es ebenfalls nicht gibt, hätte nicht dieselbe Bedeutung wie *diablare, denn *diavolare könnte wie das entsprechende *to devil nicht bedeuten '"Diable!" sagen'. Aber ein *diavolare könnte es natürlich geben, und zwar mit der Bedeutung "'Diavolo!" sagen', denn Diavolo! gibt es im Italienischen als Fluchformel. Genau dies ist nun eine der Eigenschaften delokutiver Verben, die Benveniste herausstellt: sie beziehen sich stets auf ein Sagen, auf ein Etwas-Äußern. Das Verb fringsen etwa ist insofern kein delokutives Verb. Es ist abgeleitet von einem Eigennamen und bedeutet nicht etwa '"Frings" sagen', sondern 'ein Verhalten zeigen, das Frings abgesegnet hat' und durch Generalisierung 'stehlen'.

Die zweite relevante Eigenschaft delokutiver Verben betrifft das, was gesagt, was geäußert wird. Was durch das Beispiel aus Sterne ganz deutlich wird, weil zwei Sprachen im Spiel sind, ist, dass die Basis ein Zitat ist. Auf den ersten Blick scheint es so zu sein, dass man mit einem delokutiven Verb alles Mögliche zitieren kann, z. B. Wir werden Weltmeister! mit Der wir-werden-Weltmeister-te da vielleicht herum. Aber ein Verb wie wir-werden-Weltmeister- $n$ hat sicherlich wenig Chancen ins Lexikon der deutschen Sprache einzugehen. Delokutive Wortbildungsprozesse können also in einigen Sprachen recht produktiv sein, aber für die Lexikalisierung gibt es offenbar starke Beschränkungen.

Ein lexikalisiertes delokutives Verb ist z. B. das lateinische salutare ('grüßen'). Salutare ist aus salus abgeleitet, aber nicht aus dem bloßen Substantiv, das 'Gesundheit' bedeutet, sondern aus der Grußformel Salus! ('Salut!', 'Hallo!'). Salutare heißt also ursprünglich "'Salus!" sagen'. Die Grußformel selber ist natürlich aus dem Substantiv mit der Bedeutung 'Gesundheit' abgeleitet. Dieses Beispiel lässt vermuten, dass geeignete Basen für delokutive Verben vor allem Ausrufe sind. So beschreibt Foscolo ja auch das französische Diable!. Aber mal ganz abgesehen davon, daß unter "Ausruf" ganz unterschiedliche Dinge gefasst wur- 
den und werden, gibt es doch auch Basen delokutiver Verben, die sicherlich in einem intuitiven Verständnis dieses Wortes - keine typischen Ausrufe sind, wie z. B. nec ('nicht', 'und nicht') in dem lateinischen Verb negare für 'verneinen', 'nein sagen' (!). Was nec und Salus! gemeinsam haben, ist eher, dass es sich um Ausdrücke handelt, mit denen man bestimmte ganz alltägliche sprachliche Handlungen ausführt. Man verneint bzw. grüßt. Auch das Wort für 'danken' ist nicht selten ein delokutives Verb. So bedeutet das französische Verb dafür, also remercier, ursprünglich einfach nur '"merci" sagen' (altfranzösisch noch mercier). Wäre remercier aus dem Substantiv merci ('Gunst', 'Gnade') abgeleitet, müsste es soviel bedeuten wie 'begünstigen', 'eine Gnade erweisen'.

Die sprachlichen Handlungen, die mit solchen Formeln ausgeführt werden, sind sogenannte illokutionäre Handlungen im Sinne Austins, dem bekannten Vertreter der Philosophie der normalen Sprache. Benveniste spricht im allgemeinen nicht von "Formeln", sondern von "locutions de discours", also Lokutionen mit für den Diskurs, die Rede typischen Funktionen. Der Terminus "locution" ist insofern ganz treffend, als er mehr umfasst als nur den Bezug auf feste, formelhafte Wendungen. Er spielt auch auf den Zitatcharakter an, den die Ausdrücke als Basen delokutiver Verben haben, denn zitiert wird immer die lautliche Realisierung eines Ausdrucks, und das nennt Austin den "locutionary act". Dem entspricht im Französischen der acte locutionnaire oder acte locutif. Benveniste, der mit Austins Denken sehr früh vertraut war, spielt mit dem Terminus "locution" sehr wahrscheinlich gerade auf die Terminologie Austins an.

Der Terminus "locution" ist auch insofern ganz glücklich gewählt, als er syntaktisch komplexe Ausdrücke mitumfasst. Eine syntaktisch komplexe Lokution als Basis eines delokutiven Verbs finden wir wiederum im genannten Werk von Sterne:

(4) 'tis more blasphemously sacré Dieu'd there than in any other aperture of the whole city (Sterne 1948: 175) ('dort wird blasphemischer sacré-Dieu-t als in irgendeinem anderen Teil der ganzen Stadt')

Die Lokution ist hier Sacré Dieu! als Basis einesVerbs *to sacré Dieu, dessen Grundbedeutung '"Sacré Dieu" sagen' ist. Rein formal könnte man diese Verben auch als deinterjektional behandeln, also aus Ausdrücken der syntaktischen Kategorie der Interjektionen abgeleitet.

Sogar aus Sätzen abgeleitete Lokutionen findet man als Basis lexikalisierter delokutiver Ausdrücke, wie etwa Je m'en fous! als Basis von je-m'en-foutisme. Je m'en fous! bedeutet einigermaßen harmlos übersetzt 'Das ist mir schnuppe.' Es finden sich also auch Bildungen, die keine Verben, sondern Substantive sind, sodass es auch auch delokutive Substantive gibt. Der Je-m'en-foutisme ist die Haltung, die Einstellung einer Person, die "Je m'en fous!" sagt, also Gleichgültigkeit ('Ist-mir-schnupp-ismus', 'Ist-mir-schnupp-igkeit'). Wie etwa zu com- 
munisme das Adjektiv communiste existiert, das auch substantivisch verwendet werden kann, gibt es zu je-m'en-foutisme, das ebenfalls substantivisch verwendbare je-m'en-foutiste. Varianten sind jeweils je-m'en-fichisme und je-m'enfichiste, die auf die Lokution Je m'en fiche! zurückgehen. Das Italienische kennt interessanterweise die ganz parallel konstruierten Wörter, nämlich menefreghismo und menefreghista mit der Lokution Me ne frego! als Basis. Es gibt sogar auch delokutive Substantive ohne Ableitungssuffix, z. B. frz. vasistas ('Luke') (< dt. Was ist das?), nuit-grave ('Zigarette' in der Jugendsprache) $(<$ Nuit gravement à la santé (Warnung vor den Gefahren des Rauchens auf Zigarettenschachteln)).

Auch auf Adverbien und Pronomen gehen Lokutionen zurück, die die Basis von Verben bilden, z. B. engl. to encore, frz. bisser ('Zugabe fordern'), tutoyer ('duzen') oder vouvoyer ('siezen'). Adverbien finden sich aber auch als Resultate delokutiver Ableitungen, z. B. im Französischen das veraltete fichtrement, das aus Fichtre! abgeleitet ist. Fichtre! ist sozusagen von Hause aus eine Lokution. Sie bringt Erstaunen, Bewunderung, aber auch Ärger zum Ausdruck und lässt sich im Deutschen vielleicht am besten mit Verflixt! wiedergeben. Fichtrement bon heißt dann soviel wie 'verflixt gut', 'verdammt gut'. Die ursprüngliche Bedeutung, die also, die der delokutiven Struktur entspricht, ist 'so gut, dass man "Fichtre!" sagen könnte', und die abgeleitete Bedeutung 'erstaunlich gut', 'bewundernswert gut'. Heute hört man statt fichtrement eher vachement oder diablement. Diablement ist natürlich ein von der schon erwähnten Lokution Diable! abgeleitetes Adverb, hier allerdings nicht in seinem Gebrauch als Fluchformel, sondern in seiner Verwendung als Ausdruck des Überraschtseins oder der Bewunderung. Solche Adverbien können sogar als Quantoren vorkommen, z. B. in diablement d'argent ('verteufelt/teuflisch viel Geld').

Vachement geht nicht auf eine Lokution *Vache! zurück und schon gar nicht auf vache ('Kuh') oder das Adjektiv vache ('gemein'), sondern auf die Lokution La vache!, basiert also auf einem komplexen nominalen Ausdruck. Man sieht an diesem Beispiel, dass es auch vorkommen kann, dass es Formunterschiede zwischen der Lokution und ihrem Zitat innerhalb des delokutiven Adverbs gibt. Auch La vache! dient dem Ausdruck des Erstauntseins, der Bewunderung, aber auch der Entrüstung. Es wird so deutlich, dass es hier, was die Bedeutungen delokutiver Bildungen angeht, nicht mehr um illokutionäre Handlungen geht, wie bei den oben genannten Beispielen negare und remercier etwa, sondern um Sprechereinstellungen. Auch delokutive Bildungen, die auf Lokutionen beruhen, mit denen man Einstellungen, Haltungen zum Ausdruck bringt, haben also gute Chancen lexikalisiert zu werden.

Zusammenfassend lässt sich sagen, dass Delokutivität - zumindest in manchen Sprachen - ein im Sprachgebrauch extrem produktives Verfahren ist, eine 
Äußerung durch Integration in ein Wort wiederzugeben. Es handelt sich also um eine Form der Redewiedergabe. Die Resultate delokutiver Ableitung sind teilweise auch ins Lexikon eingegangen, lexikalisiert worden. Für eine solche Lexikalisierung gibt es im wesentlichen zwei Beschränkungen, nämlich, dass die Lokutionen zu Formeln erstarrt sind und der Realisierung illokutionärer Handlungen oder dem Ausdruck von Sprechereinstellungen dienen. Man erkennt so, wie sich in der Delokutivität die Eigenschaft natürlicher Sprachen, offene Systeme zu sein, widerspiegelt, d.h. wie System und Gebrauch interagieren. Das System gestattet es einerseits, bestimmte Bildungen, die völlig neu sind, zu interpretieren, und andererseits absorbiert, mechanisiert es diese Bildungen, allerdings nur zum Teil.

\section{Possessive und Verwandtschaftsbezeichnungen}

Es gibt nun, wie einleitend gesagt, in der Syntax der italienischen Possessive ein m.W. nie gelöstes Problem, das, so werde ich zeigen, mit Hilfe des Konzepts der Delokutivität gelöst werden kann. Es geht darum, dass ein Ausdruck, der aus einem Possessiv und einer der unter (5) genannten Verwandtschaftsbezeichnungen besteht, nicht mit dem bestimmten Artikel kombiniert werden darf:

(5) padre ('Vater'), madre ('Mutter'); [babbo ('Papa'), papà ('Papa'); mamma ('Mama')] figlio ('Sohn'), figlia ('Tochter'); fratello ('Bruder'), sorella ('Schwester'); zio ('Onkel'), zia ('Tante'); nonno ('Großvater'), nonna ('Großmutter'); cugino ('Cousin'), cugina ('Cousine'); nipote ('Neffe'; 'Nichte'); marito ('Ehemann'), moglie ('Ehefrau'); suocero ('Schwiegervater'), suocera ('Schwiegermutter'); cognato ('Schwager'), cognata ('Schwägerin'); genero ('Schwiegersohn'), nuora ('Schwiegertochter')

Wir haben also als syntaktisch wohlgeformt Ausdrücke wie etwa:

(6) la mia macchina ('mein Auto'), il mio asciugamano ('mein Handtuch'); mio padre ('mein Vater'), tua zia ('deine Tante')

Nicht wohlgeformt sind dagegen:

(7) *mia macchina, *mio asciugamano; *il mio padre (in der Bedeutung 'leiblicher Vater'), *la tua zia

Es gibt allerdings dialektale Varianten mit bestimmtem Artikel, vor allem in den nördlichen Randzonen Italiens (Hölker 1996: 215-228).

Man hat nun ganz unterschiedliche Erkärungsversuche für dieses Fehlen des bestimmten Artikels bei Verwandtschaftsbezeichnungen mit Possessiven geliefert. Otto Jespersen schreibt etwa in seiner Philosophy of Grammar von 1924, der bestimmte Artikel sei bei padre und madre überflüssig, weil ja klar sei, dass man nur einen Vater und eine Mutter hat. Diese Fälle, also padre und madre hätten dann analogisch auf die anderen, also etwa figlio/figlia gewirkt. (Jespersen 1924: 111) Es gibt jedoch Umgebungen der Verwandtschaftsbezeich- 
nungen, in denen der bestimmte Artikel in Verbindung mit einem Possessiv vorkommen muss, und diese Fälle lassen sich mit Jespersens Erklärung nicht erfassen:

(8)

1. Das Possessiv ist loro: il loro padre ('ihr Vater'), nicht *loro padre

2. Das Substantiv ist pluralisch: le nostre sorelle ('unsere Schwestern'), nicht: *nostre sorelle

3. Das Possessiv steht hinter dem Substantiv: la madre nostra ('unsere Mutter'), nicht: *madre nostra

4. Das Substantiv hat ein Diminutiv-, Augmentativ- oder Pejorativsuffix: la sua zietta ('sein Tantchen'), nicht: *sua zietta

5. Das Substantiv ist Skopus eines adjektivischen Ausdrucks, eines Modfikators: $i l$ vostro caro cugino ('euer lieber Cousin'), nicht: *vostro caro cugino

6. Das Substantiv bezeichnet zwar Verwandte, liefert aber mehr Information als bloß die Information über die Verwandtschaftsbeziehung: il nostro piccolo ('unser Kleiner'), la tua primogenita ('deine Erstgeborene'), nicht: *nostro piccolo, *tua primogenita

Warum sollte nun aber eine analogische Übertragung von padre, madre auf die anderen Verwandtschaftsbezeichnungen durch diese Bedingungen eingeschränkt sein? Betrachten wir etwa die dritte Bedingung: warum keine analogische Übertragung von nostra madre auf * madre nostra?

Die traditionelle Erklärung für das Fehlen des bestimmten Artikels vor Possessiven mit Verwandtschaftsbezeichnungen stammt von dem Romanisten Wilhelm Meyer-Lübke, und findet sich im dritten Band seiner Grammatik der romanischen Sprachen aus dem Jahr 1899. Alle bedeutenden Romanisten haben sie aufgegriffen. So schreibt etwa Adolf Zauner 1926 im zweiten Band seiner Romanischen Sprachwissenschaft:

Bloß bei Verwandtschaftsnamen fehlt im Italienischen der Artikel: mio padre, mia sorella; $[\ldots]$ Ursache dieser Sonderstellung der Verwandtschaftsnamen ist deren häufige Verwendung im Vokativ, bei dem natürlich der Artikel entfällt. (Zauner 1926: 84)

Auch Heinrich Lausberg schließt sich im dritten Band seiner Romanischen Sprachwissenschaft von 1972 - wenn auch etwas zurückhaltend - dieser Auffassung an, wenn er schreibt: "Die Sonderstellung der Verwandtschaftsnamen ist wohl durch ihre häufige vokativische Funktion bedingt." (Lausberg 1972: 169) Was ist gemeint? Betrachten wir dazu folgendes Beispiel aus Italo Calvinos Fiabe italiane:

(9)Il Re aveva tre figli; li chiamò e disse: - Figli miei, mi volete bene? - Come alla nostra stessa vita, padre, - dissero i figli. 
(Calvino 1993: 985)

('Der König hatte drei Söhne; er rief sie zu sich und sagte: "Meine Söhne! Liebt ihr mich?" "So wie wir unser Leben lieben, Vater!" sagten die Söhne.')

Hier wird die Verwandtschaftsbezeichnung padre im Sinne von Zauner und Lausberg vokativisch verwendet oder anders: padre wird hier als Anredeformel oder allokutiv verwendet.

Diese Vokativhypothese lässt nun aber offen, warum Eigennamen, die ja auch als Allokutive verwendet werden können, in Verbindung mit einem Possessiv einen Artikel verlangen (Der Eigenname ist hier Zizola.):

(10) Appena gli avevano portato i vassoi, chiudeva l'uscio a chiave, faceva uscire la sua Zizola e mangiavano insieme con gran gioia. (Calvino 1993: 307)

('Kaum hatten sie ihm die Tabletts gebracht, schloss er die Tür ab, ließ seine Zizola heraus und dann aßen sie zusammen mit großem Genuss.')

Darüber hinaus kann die Vokativhypothese die sechs Ausnahmefälle unter (8) nicht erklären, also die Fälle, in denen doch der bestimmte Artikel verwendet werden muss. Und schließlich bleibt überhaupt ganz generell unklar, welcher Zusammenhang denn zwischen dem allokutiven Gebrauch einer Verwandtschaftsbezeichnung und dem Fehlen des bestimmten Artikels besteht, wenn sie zusammen mit einem Possessiv vorkommt.

Was man nun in der romanistischen Tradition offenbar nicht gesehen hat, ist, dass für die Beantwortung der genannten Fragen bestimmte Konstruktionen des Alttoskanischen relevant sind. Es gab im Alttoskanischen wie schon im Vulgärlatein Kombinationen von Verwandtschaftsbezeichnungen mit enklitischen Possessiven. Enklitische Wörter sind nachgestellte Klitika, also Wörter, die sich mit anderen Wörtern zu einer lautlichen Einheit verbinden, d.h. mit ihnen, phonetisch gesehen, ein neues Wort bilden. Das Besondere an ihnen ist, dass sie nicht den Wortakzent tragen und auch sonst nicht akzentuiert werden können. Man findet in Dante und Boccaccio etwa:

(11) mogliema ('meine Frau'), fratelto ('dein Bruder'), paremo ('mein Vater'), mammata ('deine Mama')

Diese Klitika haben sich aus den lateinischen nichtklitischen Formen meum, meam, tuum, tuam durch Lautreduktion in unakzentuierter Position entwickelt. Die italienischen Possessive mio, tuo, suo, nostro, vostro, loro sind keine Klitika. Auch diese haben sich aus den lateinischen Possessiven entwickelt. Das Interessante ist nun, dass die klitischen Possessive des Alttoskanischen nie mit einem Artikel, ja mit keinem Determinator kombiniert werden durften. Es herrscht ferner exakte Parallelität zwischen dem Gebrauch der enklitischen Possessive bei Verwandtschaftsbezeichnungen im Alttoskanischen und den unter (8) genannten Restriktionen für das Fehlen des bestimmten Artikels bei Possessiven 
und Verwandtschaftsbezeichnungen im heutigen Standard (Hölker 1998: 569-571):

(12)

1. Loro ersetzte die enklitischen Possessive der dritten Person Plural so und $s a$. Es war urprünglich kein Possessiv, sondern der Genitiv Plural von lat. ille, war also ein adjektivischer Ausdruck. Adjektivische Ausdrücke verlangten aber den bestimmten Artikel oder sonst einen Determinator.

2. Pluralische enklitische Possessive gab es im Alttoskanischen nicht, also mussten die nichtklitischen verwendet werden und diese verlangten vor allem in einer etwas späteren Entwicklungsphase des Italienischen den bestimmten Artikel oder sonst einen Determinator.

3. Ein nichtklitisches Possessiv wurde hinter einer Verwandtschaftsbezeichnung verwendet, um kontrastiv zu akzentuieren: La madre nostra, non la madre vostra. Dort konnte dann kein klitisches Possessiv mehr vorkommen.

4. Eine Verwandtschaftsbezeichnung mit Diminutiv-, Augmentativ- oder Pejorativsuffix konnte nicht mit einem enklitischen Possessiv kombiniert werden. Also mussten die nichtklitischen verwendet werden.

5. Auch wenn die Verwandtschaftsbezeichnung Skopus eines adjektivischen Ausdrucks, also syntaktisch modifiziert war, konnte sie sich nicht mit einem enklitischen Possessiv verbinden.

6. Es gab keine Kombinationen von enklitischen Possessiven mit Verwandtschaftsbezeichnungen im weiteren Sinne, also etwa piccolo/piccola, primogenito/primogenita.

Es zeigt sich so die Parallelität, um nicht zu sagen Isomorphie zwischen den Bedingungen für das Fehlen des bestimmten Artikels bei Verwandtschaftsbezeichnungen mit Possessiven und den Bedingungen für das Vorkommen der enklitischen Possessive bei den Verwandtschaftsbezeichnungen im Alttoskanischen.

Die enklitischen Possessive verschwanden nach und nach aus dem Toskanischen. Sie wurden durch die nichtklitischen verdrängt. Dies gilt jedenfalls für das gehobenere Toskanisch. Im familiären Sprachgebrauch finden sich die enklitischen Possessive mit Verwandtschaftsbezeichnungen noch bis ins 18. Jh. Ende des 16. Jh. ist der Verdrängungsprozess aus dem gehobeneren Toskanisch definitiv abgeschlossen, wie Castellani Pollidori (1967-1970: 93) mit einem Zitat aus Benedetto Varchis L'Ercolano von 1570 zeigt. Sie fasst die Entwicklung folgendermaßen zusammen: der Gebrauch der klitischen Possessive bei Verwandtschaftsbezeichnungen war in der Toskana des 16. Jh. noch ziemlich lebendig, befand sich aber schon auf dem Rückzug. Für das 17. Jh. werden die Belege immer spärlicher und im Laufe des 18. Jh. gewinnt die Verwendung der nichtklitischen Possessive auch bei Verwandtschaftsbezeichnungen die Oberhand, bei denen aber weiterhin der bestimmte Artikel fehlt. (Castellani Pollidori 1967-1970: 96) 
Das System der Possessive verändert sich also völlig, wobei nur ihre Syntax bei Verwandtschaftsbezeichnungen einigermaßen stabil bleibt. Es erhebt sich somit die Frage, was denn an Verwandtschaftsbezeichnungen so besonders ist, dass sie in dieser massiven Umgestaltung des Possessivsystems eine so spezielle Rolle gespielt haben.

Nun, die Antwort lässt sich jetzt erahnen: Verwandtschaftsbezeichnungen haben einen delokutiven Gebrauch. Betrachten wir dies ein bisschen genauer. Verwandtschaftsbezeichnungen spielen eine Doppelrolle. Sie können deskriptivreferentiell verwendet werden, wie etwa in Vater werden ist nicht schwer, Vater sein dagegen sehr oder auch als Anredeformeln, als Lokutionen also, wie etwa in Vater! Komm' mal!. Nun gibt es im Italienischen, wie auch in anderen Sprachen, erhebliche Beschränkungen für den deskriptiv-referentiellen Gebrauch von Verwandtschaftsbezeichnungen (Renzi 1991: 398-399). Man kann z. B. zu einem Kind sagen:

(13) Vieni (va) da papà! ('Komm' (geh') zu Papa!')

Papà wird hier ohne den bestimmten Artikel verwendet. Die Person, auf die man sich mit so verwendeten Verwandtschaftsbezeichnungen bezieht, muss ein Verwandter / eine Verwandte des Sprechers / der Sprecherin sein oder ein Verwandter / eine Verwandte des Adressaten / der Adressatin. Diese Beschränkung gibt es für den ursprünglichen, normalen Gebrauch der Verwandtschaftsbezeichnungen wie etwa in essere come un padre natürlich nicht. Allein die Tatsache, dass es so starke Beschränkungen gibt, ist ein Hinweis darauf, dass man es in Fällen wie (13) mit einem abgeleiteten deskriptiv-referentiellen Gebrauch von Verwandtschaftsbezeichnungen zu tun hat. Das Fehlen des Artikels wie beim Gebrauch als Anredeformel ist ein Hinweis darauf, dass dieser deskriptiv-referentielle Gebrauch aus dem Gebrauch als Anredeformel abgeleitet ist, dass so ein Ausdruck wie papà in (13) also delokutiv ist. Er ist aus der Anredeformel Papà! abgeleitet. Die Bedeutung von (13) lässt sich somit folgendermaßen umschreiben:

(14) Komm' (geh') zu der Person, die du mit "Papà!" anredest!

Ein weiterer Hinweis darauf, dass es hier um delokutive Verwendungen geht, ist, dass pluralische Verwandtschaftsbezeichnungen so nicht vorkommen können. Ich kann zwar sagen:

(15) Non fare i dispetti a nonno! ('Sei artig zu Großvater!') aber nicht:

(16) *Non fare i dispetti a nonni! (''Sei artig zu Großeltern!')

sondern nur:

(17) Non fare i dispetti ai nonni! ('Sei artig zu den Großeltern!') 
Man muss im Plural also den bestimmte Artikel verwenden.

Es ist also klar, dass Verwandtschaftsbezeichnungen völlig unabhängig von ihrem Vorkommen in Kombination mit Possessiven einen delokutiven Gebrauch aufweisen. Man kann daher annehmen, dass die Kombinationen von Verwandtschaftsbezeichnungen mit enklitischen Possessiven im Alttoskanischen delokutive Bildungen waren. Das alttoskanische mogliema unter (11) z. B. bedeutete also ursprünglich 'die Person, die ich mit "Moglie!" anrede'. Als stablisierender Faktor, der zum Erhalt der Beschränkungen für den Artikelgebrauch beim Umbau des Possessivsystems geführt hat, lässt sich so die Delokutivität solcher Konstruktionen ausmachen.

Was die Erklärung des Artikelgebrauchs bei Verwandschaftsbezeichnungen und Possessiven über Delokutivität besonders plausibel macht, ist, dass das Altportugiesische und das moderne Brasilianisch dieselben Strukturen aufweisen wie das Italienische:

(18) o meu passaporte ('mein Pass'); meu pai ('mein Vater')

und ferner, dass enklitische Possessive noch heute im Schriftkatalanischen und dialektalen Rumänisch bei Verwandtschaftschaftsbezeichnungen und nur bei diesen Verwendung finden:

(19) Katalanisch: el meu cavall ('mein Pferd'), mon pare ('mein Vater'); Rumänisch: fratele t_u ('dein Bruder'), frate-tu ('dein Bruder')

Dies lässt darauf schließen, das die entsprechenden Konstruktionen aus Verwandtschaftsbezeichnung mit enklitischem Possessiv des Vulgärlateins als delokutive entstanden sind. Solche Ausdrücke sind belegt:

(20) pater mus ('mein Vater'), mater ma ('meine Mutter') (Briefe eines Soldaten des Trajan, 2. Jh. n. Chr.)

Diese delokutive Interpretation ist dann im Laufe der Sprachgeschichte immer mehr verblasst, sodass uns diese Ausdrücke heute als etwas Unregelmäßiges erscheinen.

Was nun den heutigen Gebrauch des bestimmten Artikels in Kombination mit Possessiven und Verwandtschaftsbezeichnungen betrifft, gilt ebenfalls, dass ihr delokutiver Charakter intuitiv kaum noch erfassbar ist, weil viele Verwandtschaftsbezeichnungen nicht mehr allokutiv verwendet werden können. Den einleitend erwähnten Ausdruck mio marito wird heute niemand mehr im Sinne von 'die Person, die ich mit "Marito!" anspreche' verstehen, denn ihren Mann spricht heute niemand mehr mit Marito! an oder den Bruder mit Fratello!. Dies war jedoch mit allen Verwandtschaftsbezeichnungen noch bis in 19. Jh. hinein üblich, wovon man sich z. B. anhand italienischer Märchen schnell überzeugen kann:

(21) Aiuto! Sorella! (Calvino 1993: 224) ('Hilfe! Schwester!') 
Das Phänomen, dass delokutive Ausdrücke als solche nicht mehr transparent sind, findet man auch bei anderen delokutiven Bildungen. Man braucht gar nicht so extreme Fälle wie etwa frz. bigot zu betrachten. Wer empfindet etwa heute noch frz. pester ('fluchen') als mit peste zusammenhängend ('"Peste!" sagen')? Prinzipiell ist ein solcher Zusammenhang natürlich noch erkennbar. Das Adjektiv bigot ('frömmelnd', 'scheinheilig', 'bigott') geht dagegen auf ein normannisches Bi God! zurück. Die Normannen wurden also von den Romanen als (bloße) "'Bi God!'-Sager" bezeichnet. Insofern kann die heute fehlende Transparenz bezüglich Delokutivität - dasselbe gilt in Bezug auf den Vokativ für die Vokativhypothese - nicht als Argument gegen die hier entwickelte Delokutivitätshypothese gewendet werden.

Ein weiterer Faktor, der zu den hier besprochenen besonderen Konstruktionen bei Verwandtschaftsbezeichnungen geführt hat, ist sicherlich auch der formelhafte Charakter der erwähnten Konstruktionen mit enklitischen Possessiven. Dazu sei verwiesen auf Castellani Pollidori (1967-1970: 86) und Hölker 1996: 231-237; 1998: 574).

\section{Literaturangaben}

Benveniste, Emile (1966[1958]): "Les verbes délocutifs". Problèmes de linguistique générale. Bd. 1. Paris: 277-288.

Calvino, Italo (1993 [1956]): Fiabe italiane. Mailand.

Castellani Pollidori, Ornella (1967-1970): "Ricerche sui costrutti col possessivo in italian". Studi linguistici italiani 7: 37-98.

Conte, Maria-Elisabeth (1984): "Délocutivité, performativité, contreperformativité". In: Serbat, Guy (ed.): E. Benveniste aujourd'hui. Bd. 1. Paris: 65-76.

Hölker, Klaus (1996): Die Possessive des Italienischen. Münster.

Hölker, Klaus (1998): "Un caso di delocutività: l'assenza dell'articolo davanti al possessivo con nome di parentela in italiano (e altre lingue romanze)". In: Ramat, Paolo/ Roma, Elisa (Hrgs.): Sintassi storica. Rom: 567-576.

Jespersen, Otto $\left({ }^{10} 1968\right.$ [1924]): The Philosophy of Grammar. London/New York.

Lausberg, Heinrich (1972): Romanische Sprachwissenschaft. Bd. 3. Berlin/New York.

Meyer-Lübke, Wilhelm (1899): Grammatik der romanischen Sprachen. Bd. 3. Leipzig.

Renzi, Lorenzo ( ${ }^{3} 1991$ [ [1988]): Grande grammatica italiana di consultazione. Bd. 1. Bologna.

Sterne, Laurence (1948 [1768]): A Sentimental Journey through France and Italy. London.

Sterne, Laurence (1970 [1813]): Viaggio sentimentale di Yorick lungo la Francia e l'Italia. Mailand.

Zauner, Adolf $\left({ }^{4} 1926\right)$ : Romanische Sprachwissenschaft. Bd. 2. Berlin/Leipzig. 